\title{
CAMARADAS DISTANTES: LA RELACIÓN ENTRE CHINA Y LA REPÚBLICA POPULAR DEMOCRÁTICA DE COREA EN LA ERA DEL AUGE NUCLEAR NORCOREANO
}

\author{
Gabriela Guadalupe Márdero Jiménez
}

\section{INTRODUCCIÓN}

Se atribuye al dirigente chino Mao Zedong haber dicho que su país y la República Popular Democrática de Corea (en adelante, Corea del Norte o RPDC) eran "tan cercanos como los labios y los dientes". El discurso norcoreano sobre la relación con China también habla de una cercanía no sólo determinada por la geografía, sino fortalecida por la historia y la ideología, al haber sido "forjada con sangre en la guerra victoriosa para liberar a la patria”, como solía decir el primer líder norcoreano Kim Il-sung.

Chinos y coreanos pelearon juntos contra los invasores japoneses en los años treinta del siglo pasado y los segundos apoyaron a los comunistas chinos durante la guerra civil que libraron contra los nacionalistas, que terminó con el triunfo comunista en octubre de 1949. Encumbrado en el poder desde esa fecha, Mao Zedong movilizó tropas y equipo para apoyar a la recién creada RPDC de Kim Il-sung durante la Guerra de Corea (1950-1953). China contribuyó, más que ningún otro país, a la reconstrucción norcoreana tras la firma del armisticio que puso fin a los enfrentamientos entre el norte y el sur de la península coreana en 1953.

Desde entonces, China ha seguido apoyando económica, política, humanitaria y diplomáticamente a los líderes norcoreanos que se fueron sucediendo: Kim Il-sung (en el poder, de 1948 a 
1994), su hijo Kim Jong-il (finales de 1994 a 2011) y su nieto Kim Jong-un (que ascendió al poder a finales de 2011 y continúa en él hasta la fecha).

A lo largo de estas décadas, China ha sido prácticamente el único socio comercial y aliado político de un vecino misterioso y aislado internacionalmente. Hoy, Corea del Norte depende casi en su totalidad de China para cubrir sus necesidades o de combustible o de alimentos y medicinas o de rutas comerciales. China, a su vez, ha tenido en la RPDC un aliado en la compleja trama de las relaciones regionales en el noreste de Asia, y un amortiguador entre su territorio y Corea del Sur, país aliado de Estados Unidos, según un tratado de defensa en vigor, en donde se encuentran actualmente cerca de 30 mil soldados estadounidenses. Pese a la cercanía que los gobiernos chino y norcoreano han mantenido a lo largo de seis décadas, las relaciones entre ambos países han sido inusualmente pobres en los últimos años y el diálogo político ha ido decayendo.

Desde sus respectivos ascensos al poder, el actual líder norcoreano Kim Jong-un (diciembre de 2011) y el presidente chino Xi Jinping (noviembre de 2012) no han sostenido aún un encuentro bilateral, cuando en el pasado, por ejemplo, Kim Jong-il, padre de aquél, viajó tres veces a China tan sólo entre 2010 y 2011 para reunirse con el liderazgo político y es de todos conocida la buena relación que sostuvo, particularmente hacia el final de su vida, con el entonces presidente chino $\mathrm{Hu}$ Jintao.

Es fama que en Beijing el actual liderazgo político chino no siente gran simpatía por el joven dirigente norcoreano, como ha quedado demostrado no sólo con la falta de concreción de un encuentro bilateral entre sus líderes, sino en la escasez de contactos políticos de alto nivel y en la ausencia de representantes chinos en eventos partidistas norcoreanos, entre otros hechos que se revisarán líneas abajo.

El aparente alejamiento actual entre Corea del Norte y China está ligado a dos grandes procesos ocurridos al interior de cada uno de estos países en las últimas décadas. Por un lado, el desarrollo de su programa nuclear y de misiles balísticos, con el propósito de defenderse de una posible invasión estadounidense y 
de disuadir a su vecino del sur de intentar reemplazar al gobierno y reunificar la península bajo su control. El estatus de Estado con capacidad nuclear que Corea del Norte actualmente ostenta, aunado a su agresiva retórica contra Corea del Sur, Japón y Estados Unidos, ha alarmado a la comunidad internacional y ha convertido a ese país en una fuente de inestabilidad regional e internacional. Por otro, en los últimos años China ha logrado convertirse en la segunda economía mundial y está desarrollando una activa política exterior como parte de su estrategia para alcanzar, en el escenario internacional, un lugar más acorde a su fortaleza actual. En la revigorizada política exterior china, la estabilidad regional y la contribución al reforzamiento del multilateralismo son piezas clave.

Hay en la comunidad internacional la percepción de que China no está haciendo todo lo que está a su alcance para lograr un cambio en la postura de Kim Jong-un respecto a sus amenazas nucleares, ni para implementar a cabalidad, en su calidad de principal socio comercial de Corea del Norte, las sanciones económicas y financieras que le fueron impuestas recientemente por el Consejo de Seguridad para forzarlo a frenar el desarrollo de su programa nuclear.

¿Qué detiene a China para asumir una postura más estricta con Corea del Norte y ejercer mayor presión para lograr la moderación del régimen? ¿Hasta dónde seguirá apoyando económica y diplomáticamente al régimen de los Kim? En el presente artículo, se intentará dar respuesta a estas preguntas, partiendo de la descripción de la evolución histórica de la relación sinonorcoreana y del estado actual de la misma en el entorno del internacionalmente condenado programa nuclear y de misiles norcoreano y del ascenso de China a una posición internacional de mayor liderazgo y de mayor responsabilidad con el statu quo internacional. El propósito del artículo es contribuir al conocimiento de la relación entre China y Corea del Norte, relación que trasciende la relevancia bilateral al influir en la dinámica regional y ocupar un lugar prioritario en la agenda internacional de seguridad. 
"Resist America. Aid Korea". Primeros contactos oficiales y la Guerra de Corea

La RPDC fue creada en septiembre de 1948 gracias al respaldo soviético y teniendo al frente al antiguo guerrillero de la resistencia antijaponesa, Kim Il-sung. Poco después, Mao Zedong y los comunistas anunciaron la fundación de la República Popular China (RPC) el 1 de octubre de 1949, tras su victoria en la guerra civil contra los nacionalistas del Kuomitang. Ambas repúblicas populares establecieron relaciones diplomáticas el 6 de octubre de 1949.

Corea del Norte sería uno de los primeros países en buscar establecer lazos formales, gesto ampliamente apreciado por el gobierno de Mao, pues en las décadas siguientes pocas naciones reconocieron oficialmente a la República Popular China y la mayoría conservó la relación diplomática con la República de China, exiliada en Taiwán.

Con esto se formalizaban los vínculos que el Partido Comunista Chino (PCCh) y el liderazgo norcoreano mantenían desde años atrás, que habían sido lo suficientemente estrechos como para incluir la participación de soldados coreanos en la guerra civil china para apoyar a Mao y sus camaradas del PCch. ${ }^{1}$

Esta recién establecida relación diplomática pronto tuvo su primera prueba con el inicio de la Guerra de Corea en junio de 1950, primer conflicto bélico en el contexto de la Guerra Fría que enfrentó, por un lado, a Corea del Norte con el apoyo militar de China y, en menor medida, de la Unión de Repúblicas Socialistas Soviéticas (URss) y, por el otro, a Corea del Sur respaldada por una fuerza militar multinacional bajo el mando de Estados Unidos. Al final de la Segunda Guerra Mundial la península coreana, que llevaba treinta y cinco años bajo control japonés, quedó dividida en dos zonas de ocupación: la soviética, al norte de la línea ecuatorial conocida como paralelo 38, y la estadounidense, al sur del mismo.

${ }^{1}$ Cf. A. Cathcart y Ch. Kraus; "The Bonds of Brotherhood: New Evidence on Sino-North Korean Exchanges, 1950-1954”, Journal of Cold War Studies, vol. 13, núm. 3, 2011, p. 29. 
Esta situación pronto dio paso a la creación de dos gobiernos provisionales y, posteriormente, de dos países. Estados Unidos y las Organizaciones de las Naciones Unidas (ONU) apoyaron la formación de la República de Corea, con el nacionalista cristiano Syngman Rhee como presidente, y la uRss sustentó la creación de la RPDC de Kim Il-sung.

Ninguna de las dos Coreas quedó conforme con los límites fronterizos establecidos y cada una reclamó la totalidad de la península coreana, lo que dio inicio a un periodo en que los escarceos militares a lo largo del paralelo 38 se volvieron moneda común. ${ }^{2}$ Además de soldados, en esa línea divisoria se enfrentaban dos visiones distintas del futuro de la península coreana, plasmadas en el desarrollo de dos sistemas políticos y socioeconómicos radicalmente diferentes. En el caso de la Corea al norte del paralelo 38, Kim implementó un sistema "anti-imperialista, anti-feudal y de transición al socialismo", ${ }^{3}$ que encontró en rusos y chinos una fuente de apoyo tanto ideológica como tangible. Corea del Sur nació con el apoyo de Estados Unidos y con la intención de erigirse en una democracia con un sistema económico capitalista. ${ }^{4}$

Con el propósito de unificar la península bajo el control de la RPDC, el líder norcoreano visitó tanto a Stalin como a Mao en sus respectivas capitales, solicitando apoyo para lanzar una operación militar relámpago contra Corea del Sur. Stalin se manifestó a favor de la idea, pero no se comprometió a apoyar directamente a los norcoreanos en su campaña militar. Más aún, sugirió a Kim buscar el apoyo de Mao, dado su reciente éxito en la guerra civil china y su "buen entendimiento de los asuntos de Oriente". ${ }^{5}$ Con esto, Stalin colocó sobre los hombros de China, un país de muy reciente creación y agotado por años de una larga guerra civil, buena parte de la responsabilidad de una invasión militar norcoreana que, en

${ }^{2}$ H. Kissinger, On China, Nueva York, Penguin Press, 2011, p. 123.

${ }^{3}$ Kim Il-Sung. Condensed Biography, Pyongyang, Foreign Languages Publishing House, 2001, p. 137.

${ }^{4}$ S. Hoom Kil y Chung-in Moon, Understanding Korean Politics: An Introduction, Albany, NY, State University of New York Press, 2001, pp. 9 y 79.

${ }^{5}$ S. N. Goncharov, J. Lewis y X. Litai, Uncertain Partners Stalin, Mao, and the Korean War, Stanford, University Press, 1993, p. 144. 
el contexto de la tensión de la Guerra Fría, convirtió al noreste de Asia en la primera frontera del enfrentamiento bipolar. Mao también respaldó la idea durante la visita a Beijing, en la que Kim le planteó sus proyectos, pero éste regresó a su país sin haber acordado aparentemente con Mao la realización de la invasión en una fecha concreta o mayores detalles de la misma. ${ }^{6}$

Kim invadió Corea del Sur la madrugada del 25 de junio de 1950. No se produjo una victoria relámpago como había garantizado a Stalin y a Mao, aunque el ejército norcoreano avanzó velozmente, cubriendo prácticamente la totalidad de la península, salvo por la punta sur de Busan. El presidente de Estados Unidos, Harry Truman, convocó a reunión urgente del Consejo de Seguridad de la onU, de la que emanó la aprobación para la conformación de una fuerza multinacional, comandada e integrada principalmente por Estados Unidos, para defender a Corea del Sur de la agresión norcoreana. La votación en el Consejo se realizó ante la ausencia temporal de delegados soviéticos, con lo cual se evadió el posible veto de la unss a la resolución.

La entrada de esta fuerza multinacional pronto revirtió los logros norcoreanos, haciendo retroceder a las fuerzas de Kim hasta el paralelo 38. Tras los éxitos militares de principios de su campaña en la península coreana, Truman amplió el objetivo de la misma para no únicamente hacer retroceder a los norcoreanos al paralelo 38, sino unificar la península bajo el control de Corea del Sur, idea que fue respaldada por la comunidad internacional. Al entender que esto implicaría la invasión de la totalidad del territorio norcoreano y la expansión de Corea del Sur (o sea del ejército estadounidense) a las puertas mismas del noreste chino, Mao advirtió que no aceptaría que las fuerzas de la onu cruzaran el paralelo 38. Éstas prosiguieron la ofensiva; tomaron la capital norcoreana y siguieron hasta llegar a escasos kilómetros de la frontera con China.

Mao, fiel al lema del momento, "Resistir a América y ayudar a Corea", llevaba meses desplazando equipo y hombres a la zona del Río Yalu, frontera sinonorcoreana. Los 250 mil efectivos militares

${ }^{6}$ E. Anguiano (coord.), China contemporánea. La construcción de un país (desde 1949), México, El Colegio de México, 2011, p. 200. 
chinos que se encontraban ya en el área ${ }^{7}$ fueron comandados para hacer el debut formal de China en la Guerra de Corea con un ataque que hizo retroceder a las fuerzas de la onu a Seúl. Estados Unidos respondió naval y aéreamente de inmediato, volviendo a colocar el frente de batalla en el paralelo 38. Durante varios meses las líneas de batalla se movieron hacia atrás y hacia delante del paralelo, manteniéndose en general un estancamiento militar. En esas circunstancias, el nuevo presidente estadounidense, Dwight D. Eisenhower, amenazó con hacer uso de la bomba nuclear para terminar la guerra, generando al corto plazo el inicio de conversaciones sobre un posible armisticio y, a largo, la introducción del tema nuclear en la península coreana.

Con el recuerdo fresco en la memoria de los ataques nucleares estadounidenses sobre las ciudades japonesas Hiroshima y Nagasaki pocos años atrás y tras la muerte de Stalin -quien recomendaba arrastrar las negociaciones y, en consecuencia, la guerra tanto como se pudiera-, las conversaciones derivarían en la firma de un acuerdo de armisticio, que no un tratado de paz, en julio de 1953. A partir del acuerdo, firmado en una breve ceremonia en Panmunjon, frontera de facto entre ambas Coreas en el paralelo 38, cesaron los actos armados por un tiempo indeterminado, hasta el logro de un acuerdo permanente que aún no se ha alcanzado. La frontera entre las dos Coreas quedó más o menos en el mismo sitio donde estaba tres años antes del armisticio.

Para la República Popular China, los combates representaron un esfuerzo enorme, principalmente en recursos materiales. Se estima que la ayuda brindada a Corea del Norte le costó 10000 millones de dólares, una gran suma para un país de reciente creación, que salía de una larga guerra civil. ${ }^{8}$ Sin embargo, su participación en la Guerra de Corea cumplió el propósito de constituirse en un triunfo sobre la mayor potencia mundial en una guerra convencional y le permitió el aseguramiento de su frontera, de su credibilidad militar y de su liderazgo comunista

${ }^{7}$ J. Chen, China's Road to Korean War: The Making of the Sino-American Confrontation, Nueva York, Columbia University Press, 1994, p. 144.

${ }^{8}$ Anguiano, op. cit., p. 203. 
en Asia. ${ }^{9}$ En lo que toca a la política interior, haber enfrentado a Estados Unidos sirvió al Partido Comunista para, por medio de una intensa campaña propagandística, consolidar su poder a escasos cuatro años de haber ganado la guerra civil.

Al mismo tiempo, la reticencia de Stalin a intervenir en la Guerra de Corea fue clave para el acercamiento entre Corea del Norte y China. ${ }^{10}$ La primera se alejó de la uRss para acercase a la segunda, a la cual haber apoyado a los norcoreanos durante y después de la guerra le valió una enorme capacidad de influencia en los asuntos de la península, que conserva hasta el día de hoy.

Si la ayuda militar a Corea del Norte durante la guerra marcó el inicio de la influencia china en la península, su apoyo para la reconstrucción del país, en ruinas tras la guerra, terminó de sellar la alianza. Luego de tres años de bombardeos, la producción industrial, agrícola y energética en la parte norte de la península coreana había colapsado, al igual que su infraestructura de vivienda y transportes. El gobierno norcoreano estimaba que los daños ascendían a 420 billones de wons, cerca de 170 millones de dólares según el tipo de cambio de entonces. ${ }^{11}$

En los años siguientes, China se convirtió en el mayor proveedor de los bienes de consumo que le permitieron a los norcoreanos solventar sus necesidades básicas diarias, de los materiales de construcción y la mano de obra para reconstruir el país, así como de las redes ferroviarias para construir su economía. El gobierno de Mao decidió también condonar la deuda por préstamos otorgados a Corea del Norte durante la guerra. ${ }^{12}$ China además recibió a miles de refugiados, soldados retirados y estudiantes, cuyos descendientes habitan hoy en la Prefectura Autónoma de Yanbian, región en el noreste de China, que al menos desde el siglo XII ha

${ }^{9}$ Kissinger, op. cit., p. 147.

${ }^{10}$ A. Lankov, Crisis in North Korea: The Failure of De-Stalinization, 1956, Honolulu, University of Hawaii Press, 2004, p. 19.

11 Z. Shen y Y. Xia, China and the Post-War Reconstruction of North Korea, 19531961, Washington, DC, Woodrow Wilson International Center for Scholars-North Korea International Documentation Project, 2012, p. 2.

${ }^{12} \mathrm{Ch}$. Armstrong, "«Fraternal Socialism»: The International Reconstruction of North Korea, 1953-1962”, Cold War History, vol. 5, núm. 2, 2005, p. 164. 
recibido a inmigrantes provenientes de la península, por lo cual afectuosamente se la conoce como la Tercera Corea. ${ }^{13}$

\section{DANDO FORMA Y FONDO A LA RELACIÓN BILATERAL. DEL FIN DE LA Guerra de Corea AL "AÑo de la AMistad Sinocoreana"}

La relación entre China y Corea del Norte en los años que siguieron a la firma del armisticio en Panmunjon debe entenderse a la luz de la Guerra Fría. La afinidad comunista entre ambos, en un entorno mundial dividido en dos polos ideológico y económico, no sólo los unió ante un enemigo común, Estados Unidos, sino que delineó la esencia de la relación.

En 1953, ambos países firmaron un Acuerdo de Cooperación Económica y Cultural. En su discurso durante el banquete ofrecido a la delegación norcoreana en Beijing, Mao Zedong descartó que la cooperación comprometida fuese a ser unilateralmente proporcionada por China, pues dijo que Corea había hecho ya su parte, al estar "en la primera línea de la lucha contra el imperialismo, protegiéndonos de los enemigos que querían cruzar el río Yalu". ${ }^{14}$ En los siguientes años, China apoyó la construcción y puesta en marcha de ingenios, molinos, fábricas de seda y papel, plantas generadoras de energía, granjas, presas y minas. ${ }^{15}$ Simultáneamente se llevaron a cabo visitas mutuas de importantes funcionarios del PCCh y del Partido de los Trabajadores Coreanos, así como de los respectivos ejércitos populares.

${ }^{13}$ Para un estudio detallado sobre la acogida de coreanos étnicos en la Prefectura de Yanbian tras la guerra, véase A. Cathcart y Ch. Kraus, op. cit.

${ }^{14}$ Wilson Center, "Mao Zedong's Remarks at the Banquet for the North Korean Government Delegation”, 23 de noviembre de 1953, History and Public Policy Program Digital Archive, trad. de Jeffrey Wang y Charles Kraus, en http:/ /digi talarchive.wilsoncenter.org/document/114167, consultado el 8.XII.2016.

15 Cf. Wilson Center, "Reference Materials for the Sino-Korean Negotiations on Supplying Equipment and Constructing Power Plants”, 5 de agosto de 1958, History and Public Policy Program Digital Archive, trad. de Jeffrey Wang y Charles Kraus, en http://digitalarchive.wilsoncenter.org/document/114175, consultado el 10.XII.2016. 
Para institucionalizar estos intercambios, en 1961 adoptaron el Tratado de Amistad, Cooperación y Asistencia Mutua, que se ha renovado en dos ocasiones (1981 y 2001) y deberá de refrendarse en 2021. El eje del acuerdo es la cooperación militar: con la firma del documento cada parte ofreció defender a la otra en caso de un ataque externo, además de comprometerse a consultar al otro acerca de asuntos internacionales de interés común, brindarse toda la ayuda técnica y económica posible y consolidar sus lazos culturales y científicos.

El impulso que la relación llevaba desde el establecimiento formal de vínculos diplomáticos en 1949, sellada en 1961 con la firma del Tratado de Amistad, sufrió un estancamiento durante la convulsa década de la Revolución Cultural. Durante la fase activa de la misma, de 1965 a 1969, ${ }^{16}$ China optó por la discreción en sus contactos con el exterior, llegando incluso a desconfiar de sus aliados: durante ese periodo, y en creíble consonancia con el estilo maoísta del momento, Kim Il-sung fue acusado por los Guardias Rojos chinos de traidor a la causa comunista. ${ }^{17}$

Durante la siguiente etapa de la Revolución Cultural, de 1970 a 1976, la relación de China con la RPDC tampoco fue muy intensa, pues a pesar del desorden interno imperante, China estaba muy ocupada en el ámbito internacional. Logró que le restituyeran sus plenos derechos y su asiento en la Organización de las Naciones Unidas en 1971 y estableció relaciones diplomáticas con quienes parecían enemigos irreconciliables: Japón, en 1972, y Estados Unidos, en 1979, luego de haber recibido la visita del entonces presidente Richard Nixon, en 1972; e inició, en fin, su transformación en una economía de mercado hacia 1979.18

En estas circunstancias, la relación con Pyongyang era relevante para Beijing únicamente por mantener un aliado que sirviera de

${ }^{16}$ Anguiano, op. cit., p. 234.

${ }^{17}$ Cf. Wilson Center, "Journal of Soviet Ambassador in the DPRK A.M. Puzanov for 16 June 1960", 16 de junio de 1960, History and Public Policy Program Digital Archive, trad. de Gary Goldberg, en http://digitalarchive.wilsoncenter. org/document/119419, consultado el 10.XII.2016.

18 Comité Central del Partido Comunista de China, Textos escogidos de Deng Xiaoping 1975-1982, Beijing, Ediciones en Lenguas Extranjeras, 1984, p. 331. 
amortiguador en una porción de su frontera noreste. Más aún, en 1992 China estableció relaciones diplomáticas con Corea del Sur, agriándose la relación entre Beijing y Pyongyang, que permanecieron en un estado de fría cortesía durante los siguientes diez años.

Para entonces, Kim Il-sung, fundador de la RPDC había fallecido, no sin haber dejado en el poder a su hijo Kim Jong-il, quien, en 2002, viajó a Beijing. Tras los altibajos por los que había atravesado la relación, los lazos oficiales (por ejemplo, las visitas de encumbrados funcionarios) mejoraron a partir de la visita de Kim Jong-il, así como los intercambios económicos. Para 2008, China había reemergido como el principal socio comercial de Corea del Norte. En estas renovadas circunstancias, el presidente chino $\mathrm{Hu}$ Jintao y el líder norcoreano Kim Jong-il declararon el 2009 como "Año de la Amistad” entre ambos países, en conmemoración del sexagésimo aniversario del establecimiento de relaciones diplomáticas.

En el marco de las celebraciones, el primer ministro chino Wen Jiabao, al frente de una muy importante delegación, hizo un viaje a Pyongyang, el primero de un primer ministro chino en dieciocho años. Durante la visita de Wen se refrendó el compromiso de apoyarse mutuamente, y se firmaron varios documentos, entre ellos un renovado Acuerdo de Cooperación Económica y Tecnológica. Además, Wen ofreció un paquete de ayuda económica de 50 millones de dólares. ${ }^{19}$ Los vaivenes en la relación política, provocados, la mayoría de las veces, no tanto por los norcoreanos como por los chinos, fueron compensados en el ámbito económico. China, en medio de su rápido crecimiento económico durante la década de los años ochenta, fue convirtiéndose en la principal línea de abastecimiento, que hasta la fecha mantiene a Corea del Norte en pie.

Hoy, China es el principal socio de la RPDC, cuyo comercio con el resto del mundo se ha visto severamente afectado por las sanciones comerciales y financieras impuestas por el Consejo de Seguridad de las Naciones Unidas. El 82\% de las exportaciones norcoreanas (carbón, mineral de hierro, ropa) van a China, que

19 Cf. "China Brings Lavish Gifts to N. Korea”, The Chosun Ilbo, 7 de octubre de 2009, en http://english.chosun.com/site/data/html_dir/2009/10/07/20091 00700686.html, consultado el 12.XII.2016. 
provee a su aliado con el $87 \%$ de sus importaciones (petróleo, tejidos sintéticos, aceite, maquinaria pesada, tecnología para la acuacultura, equipo para el cultivo de granos y para la extracción minera). ${ }^{20}$ La mayor parte del carbón y otros minerales que Corea del Norte exporta a otras partes del mundo (India, Pakistán, Nigeria y Brasil) ha sido resultado de su asociación con compañías chinas, que aportan la maquinaria a cambio de una parte del material extraído o manufacturado.

Asimismo, la provisión china de alimentos y energía a Corea del Norte representa dos tercios del total de la asistencia humanitaria que ese país recibe anualmente desde la década de los años noventa. ${ }^{21}$ China es también el principal proveedor de inversión extranjera directa a Corea del Norte, que, según datos del Ministerio de Comercio chino, se concentra en las manufacturas (49\%), la minería (20\%), seguido de cerca por los sectores de servicios y comercio, que han repuntado particularmente en los últimos años $(19 \%) .^{22}$

No sólo de ayuda humanitaria, intercambios comerciales e inversiones provee la RPC a la RPDC. Por años, la primera ha intentado erigirse frente a la segunda como un modelo de desarrollo, es decir una muestra de cómo una economía centralizada puede crecer sin que eso implique -al menos en el discurso- comprometer sus ideales socialistas. China ha intentado inducir en la RPDC reformas semejantes a las que ha implementado en su propio país en las últimas cuatro décadas, pero su insistencia no ha encontrado oídos muy atentos entre los norcoreanos.

A la visita del primer ministro Wen Jiabao a Pyongyang, en 2009, siguió la del ministro de defensa chino Liang Guanglie; la

${ }^{20}$ Cf. R. Hausmann et al., The Atlas Of Economic Complexity. North Korea, Harvard's Center for International Development (CID), 2014, en http://atlas.media. mit.edu/en/profile/country/prk/\#dataviva, consultado el 20.I.2017.

${ }^{21}$ S. Haggard y M. Noland, Famine in North Korea. Markets, Aid, and Reform, Nueva York, Columbia University Press, 2009, p. 124.

${ }^{22}$ Cf. S. Haggard y W. Shi, "Chinese Investment in North Korea: Some Data (Part I)”, Peterson Institute for International Studies, junio de 2014, en https:// piie.com/blogs/north-korea-witness-transformation/chinese-investment-northkorea-some-data-part-i, consultado el 22.I.2014. 
quinta y sexta visitas de Kim Jong-il a China, en 2010; y tras éstas, las del secretario de la Comisión Central de Asuntos Políticos y Jurídicos de China, Zhou Yongkang (encargado del aparato de seguridad de China y las instituciones policiales, quien purga actualmente una sentencia de cadena perpetua por cargos de corrupción), y del consejero de Estado Dai Bingguo (máximo encargado de la política exterior de China en ese momento), también en 2010.

En 2011, con motivo del quincuagésimo aniversario de la firma del Acuerdo de Cooperación de 1961, el viceministro chino Zhang Dejiang visitó Pyongyang. En su reunión con Kim Jong-il, señaló que las recientes visitas mutuas de alto nivel, incluyendo las frecuentes reuniones de Kim con el presidente chino, Hu Jintao, habían dado un gran impulso al desarrollo de los vínculos bilaterales, los cuales, dijo, atravesaban por un muy buen momento. Ese buen momento en las relaciones parece que terminó entonces. La de Zhang Dejiang sería la última reunión de alto nivel entre ambos países que se haya sostenido hasta esta fecha. El segundo Kim murió pocos meses después, y el tercero, Kim Jong-un, que asumió el poder tras la muerte de su padre, no ha sostenido un encuentro de esa índole hasta el momento.

Tomando caminos SeParados: el Reino Ermitaño se arma, el Reino del Medio se abre al mundo

En los últimos treinta y cinco años, China ha pasado de ser un país aislado y empobrecido, enclavado en la ideología socialista más ortodoxa, a ser una próspera potencia económica con cada vez mayor influencia en los asuntos internacionales. En el extremo opuesto, se encuentra Corea del Norte, estado autócrata, aislado y en precarias condiciones económicas.

\section{El Reino Ermitaño se arma}

Ha sido largo el camino que ha recorrido Corea del Norte para convertirse en un Estado con capacidad nuclear, propósito que ha 
perseguido, según las palabras de sus últimos dos líderes, para hacer frente a un posible ataque estadounidense o a una invasión surcoreana, que persigan la destrucción del régimen. La amenaza nuclear de Eisenhower durante la Guerra de Corea parece no haber sido olvidada en Pyongyang.

El programa nuclear norcoreano comenzó a finales de la década de los años cincuenta, con la suscripción de acuerdos de cooperación para el desarrollo de energía nuclear con la uRss, por lo cual ingenieros y científicos norcoreanos fueron capacitados y entrenados por contrapartes rusas. ${ }^{23}$ En esta etapa inicial, comenzó a operar el primer centro de investigación nuclear en Yongbyon, 100 kilómetros al norte de la capital norcoreana, que continúa siendo la mayor instalación nuclear del país, que proporcionaría, años después, el material fisionable para el primer ensayo nuclear en 2006. Los reactores nucleares construidos con ayuda de los soviéticos tuvieron su época de esplendor a lo largo de la década de los años ochenta.

Salvo lo anterior, se sabe poco sobre el desarrollo del programa nuclear de la RPDC en sus etapas inicial e intermedia. Por ello, causó asombro que los servicios satelitales de Estados Unidos detectaran, en 1986, pruebas con materiales sumamente explosivos y una planta de reprocesamiento de plutonio de muy reciente construcción en Yongbyon, que no había sido monitoreada internacionalmente al estar Corea del Norte suscrita al Tratado de No Proliferación de Armas Nucleares (TNP) desde 1985. ${ }^{24}$

La presión internacional no se hizo esperar y relativamente rindió frutos en 1992, cuando Corea del Norte permitió que los inspectores de la Agencia Internacional de Energía Atómica (AIEA) ingresaran en las instalaciones del reactor. La AIEA encontró discrepancias importantes entre la información oficial proporcionada por el gobierno norcoreano y sus hallazgos científicos in situ, lo que sugería que el país podría tener no sólo plutonio escondido,

${ }^{23}$ E. Chanlett-Avery (coord.), "North Korea: U.S. Relations, Nuclear Diplomacy and Internal Situation", Washington, DC, Congressional Research Service, 2016, p. 12.

${ }^{24}$ M. ElBaradei, The Age of Deception. Nuclear Diplomacy in Treacherous Times, Londres, Metropolitan Books/Henry Holt \& Company, 2011, p. 37. 
sino también un programa en marcha para utilizarlo en la producción de armamento nuclear.

Luego de infructuosos intentos por obtener de parte del gobierno norcoreano mayor acceso para comprobar sus hipótesis, la AIEA solicitó conducir "inspecciones especiales", procedimiento pocas veces iniciado por la Agencia. Corea del Norte se negó a permitirlas y la AIEA concluyó que el país no cumplía con sus obligaciones en el marco del TNP, por lo que envió el asunto al Consejo de Seguridad de las Naciones Unidas. Los norcoreanos amenazaron con retirarse del tratado, generándose así lo que se conoce como la primera crisis nuclear norcoreana.

Esta crisis fue zanjada mediante un acuerdo entre Estados Unidos y Corea del Norte en 1994, bajo el cual el primero accedía a proporcionar a la segunda dos reactores de agua ligera para producción de nucleoelectricidad e insumos de combustible, así como a normalizar sus relaciones diplomáticas. A cambio, Corea del Norte ofrecía congelar y luego desmantelar su programa nuclear de fines "duales". El acuerdo sirvió para que Corea del Norte frenara su programa de plutonio -que se creía era el único que se estaba desarrollando- de 1994 a 2002 con la supervisión de la AIEA.

Pese a que ese programa estaba en general bajo control, en 2002 la situación tuvo un giro dramático. A partir de un oscuro reporte no verificado, ${ }^{25}$ el entonces presidente de Estados Unidos, George W. Bush, acusó a Corea del Norte de estar desarrollando, en secreto, un programa de enriquecimiento de uranio, la otra vía para poder hacerse de una bomba atómica además de la ruta del reprocesamiento de plutonio.

Tras incluir a Corea del Norte como parte del "eje del mal" junto con Irak e Irán, el presidente Bush detuvo tanto el programa de entrega de combustible como la construcción de los reactores de agua ligera. En respuesta, Corea del Norte expulsó a los inspectores de la AIEA, anunció su retiro del TNP -el primer país en tomar tal decisión- y la reanudación de las operaciones de sus reactores y plantas de reprocesamiento, detenidas desde hacía ocho años.

${ }^{25}$ Para mayores detalles sobre el informe Kelly, véase $i d e m$. 
Cuatro años después, en 2006, el Reino Ermitaño llevó a cabo su primer ensayo nuclear, en Punggye-ri, al noreste del país. El ensayo fue de dimensiones bastante modestas, pero expuso una sombría realidad: una nación empobrecida y aislada, desafiante, se había unido al club nuclear.

En 2009, la RPDC detonó otro artefacto nuclear, igualmente pequeño, pero más exitoso que el de 2006, con una potencia de 2 kilotones (la bomba sobre Hiroshima tuvo una fuerza de 15), y otro más en 2013 igualmente evolucionado con respecto del ensayo previo. En 2010, Corea del Norte mostró orgullosamente a un grupo de científicos estadounidenses una planta de enriquecimiento de uranio, que se encuentra en un antiguo edificio de fabricación de combustible para reactores en Yongbyon, lo cual confirmó las sospechas de larga data sobre su capacidad al respecto. ${ }^{26}$

El cuarto ensayo nuclear ocurrió en febrero de 2016, habiéndose tratado, a decir de Corea del Norte, de una bomba de hidrógeno, aunque hay sospechas sobre la veracidad de la naturaleza de la detonación. ${ }^{27}$ En septiembre de 2016, finalmente tuvo lugar el quinto ensayo nuclear con mucha mayor potencia que ensayos anteriores $(10 \mathrm{kt}) .{ }^{28}$ En este momento, el programa nuclear norcoreano está en su apogeo. Según datos hasta enero de 2016, se sabe que el país ha producido entre 30 y 40 kilos de plutonio reprocesado, suficiente para producir al menos media docena de armas nucleares. ${ }^{29}$

Si bien la mayor preocupación de la comunidad internacional, cuando se trata de Corea del Norte, es el programa de armas

${ }^{26}$ Cf. D. E. Sanger. "North Koreans Unveil New Plant for Nuclear Use", The New York Times, 20 de noviembre de 2010, en http://www.nytimes.com/2010/11/ 21/world/asia/21intel.html, consultado el 11.X.2016.

27 Cf. World Nuclear Association, "Nuclear Proliferation Case Studies", septiembre de 2016, en http://www.world-nuclear.org/information-library/safetyand-security/non-proliferation/appendices/nuclear-proliferation-case-studies. aspx, consultado el 9.X.2016.

${ }^{28}$ Cf. V. Jackson, "Preventing Nuclear War with North Korea", Foreign Affairs, 11 de septiembre de 2016, en https:/ / www.foreignaffairs.com/articles/north-korea/2016-09-11/preventing-nuclear-war-north-korea, consultado el 10.XI.2016.

${ }^{29}$ E. Chanlett-Avery, op. cit., p. 13. 
nucleares, hay otros asuntos contenciosos no menos graves, como el programa de misiles, el tamaño del ejército convencional (compuesto por 1.2 millones de soldados y unos 600000 reservistas, de una población de poco más de 25 millones de personas, el ejército norcoreano es uno de los más grandes del mundo), la producción de armas biológicas y químicas y un terrible registro de violaciones a los derechos humanos de su población. ${ }^{30}$

El programa de misiles de mediano y largo alcance es igualmente peligroso para la estabilidad regional, pues Corea del Norte ha probado ya su capacidad de impactar con misiles a Japón y Corea del Sur, con la intención de atacar la costa oeste de Estados Unidos -meta de la que se dice ya no se encuentra muy lejos. Asimismo, se sabe que va avanzando en sus intentos por miniaturizar una ojiva nuclear que pueda montar sobre un misil balístico o uno submarino. ${ }^{31}$

Hoy el programa avanza más rápido, está menos acotado y más unido al desarrollo de misiles balísticos que en cualquier otro momento. Pyongyang no deja pasar oportunidad alguna de evidenciar que su capacidad nuclear tiene por objetivo garantizar la supervivencia del régimen y que cualquier intento por reemplazarlo o invadir el país podría derivar en un desastre nuclear.

El enorme gasto militar, efectuado por un país con escasos vínculos comerciales con el exterior, se refleja en el estado de su economía. Si bien el gobierno norcoreano no hace pública su información económica, se cree que, en 2015, su рів fue de 28 millones de dólares, con lo que se ubica en el número 208 de las economías mundiales; ${ }^{32}$ el ingreso per capita fue de 1800 dólares

${ }^{30}$ Cf. Nuclear Threat Initiative, "North Korea", reporte por país, octubre de 2016, en http://www.nti.org/learn/countries/north-korea/, consultado el 5. XII.2016.

31 Véase, respectivamente, D. Albright, "North Korea Miniturization”, U.S.Korea Institute, febrero de 2013, en http://38north.org/2013/albright021313, consultado el 21.X.2016, y "Supreme Leader oversees successful SLBM test-fire", The Pyongyang Times, 27 de agosto, Juche 105, núm. 35, 2016, p. 1.

32 Cf. The World Fact Book, CIA, "North Korea 2015", octubre de 2016, en https://www.cia.gov/library/publications/the-world-factbook/geos/kn.html, consultado el 16.XII.2016. 
ese mismo año; su economía decrece a un ritmo de - $1.1 \%$ anual; su producción industrial decayó 3.4\% en 2015 y su producción manufacturera sufrió una disminución de 3.1\%. ${ }^{33}$ Empobrecimiento, aislamiento y un enorme gasto en desarrollo militar y nuclear, hacen de Corea del Norte un país que contrasta marcadamente con su aliado y vecino, con quien cada día son más las diferencias que los puntos de encuentro.

La postura del actual gobierno chino sobre el programa nuclear norcoreano es opuesta al mismo. Por medio de comunicados y declaraciones oficiales, reforzados por editoriales en los principales periódicos chinos, el gobierno del presidente Xi Jinping ha sido claro en cuanto a que el desarrollo de armas nucleares por parte de Corea del Norte compromete la seguridad de la región y debe frenarse con la negociación y el diálogo. China hace responsable, en parte, a Corea del Norte, pero con igual frecuencia presiona a los otros actores involucrados -principalmente Estados Unidos y Corea del Sur- para que mejore el entorno de seguridad de Corea del Norte y reducir así la percepción del gobierno norcoreano de sentirse amenazado, algo que, según arguye China, es la razón por la que su vecino desea hacerse con un arsenal nuclear.

\section{El Reino del Medio asciende}

Mientras Corea del Norte se armaba y se aislaba progresivamente de la comunidad internacional, China daba pasos para abrir su economía e insertarse en los mercados globales, abrir canales de diálogo político bilateral con otros actores y desplegar una diplomacia activa en su zona geográfica natural, al igual que en África y América Latina. También temáticamente la diplomacia china se ha diversificado considerablemente en las últimas décadas, para hoy estar presente en prácticamente todos los asuntos de la agenda mundial.

33 Cf. "Trading Economics 2015, North Korea", septiembre de 2015, en http:/ / www.tradingeconomics.com/north-korea/gdp, consultado el 16.XII.2016. 
El proceso de transformación del modelo económico socialista chino inició con las reformas impulsadas por Deng Xiaping a finales de la década de los años setenta, consistentes en la apertura al comercio mundial y a las inversiones privadas extranjeras y en el fomento al surgimiento de un sector privado. ${ }^{34}$ Por la continuidad que los sucesivos liderazgos chinos han dado al proceso de apertura y modernización que inició Deng, modelo cuyos dirigentes denominan "economía socialista de mercado", el tamaño de la economía se ha cuadruplicado en las últimas dos décadas y el país se ha convertido en el mayor centro mundial de manufactura y exportaciones, ${ }^{35}$ ubicándose como la segunda economía mundial. El entorno económico chino se caracteriza actualmente por el acelerado crecimiento y diversificación, el creciente dominio de la tecnología, una posición financiera determinante para la estabilidad global del sistema y un accionar internacional asertivo que rebasa su entorno regional inmediato. ${ }^{36}$

En lo que atañe a la sociedad, China ha pasado de ser un país eminentemente rural a desarrollar gigantescos centros urbanos, proyectados para continuar expandiéndose. En el ámbito diplomático, es difícil pensar hoy en un foro, diálogo, organización o tema, en el que China no participe. Políticamente, el Estado chino es sólido y ha generado los mecanismos que le permiten mantener el control social, político y mediático, necesario para su supervivencia.

En síntesis, en las últimas cuatro décadas China se ha transformado a un ritmo más acelerado que en cualquier momento de su historia anterior. Este meteórico ascenso ha transformado la dinámica no sólo asiática, sino internacional, y el potencial del país para seguir influyendo en los temas prioritarios mundiales es muy alto. Como el gigante regional que es, y habiendo además mantenido lazos estrechos con Corea del Norte desde su fundación, China recibe presión por los cuatro costados para incidir en la moderación

${ }^{34}$ Anguiano, op. cit., p. 387.

35 A. Cheremukhin et al., The Economy of People's Republic of China from 1953, National Bureau of Economic Research, 2015.

${ }^{36}$ J. E. Navarrete, op. cit., p. 13. 
del gobierno de Pyongyang, presión que, en su calidad de nueva potencia mundial, al parecer ya no desea seguir recibiendo.

\section{RENOVACIÓN DE LOS LIDERAZGOS Y CONTACTOS (ESCASOS) ENTRE ELLOS}

Desde la llegada al poder de Kim Jong-un y Xi Jinping se percibe un enfriamiento en la relación entre ambos países. En el caso norcoreano, Kim Jong-un heredó las riendas de la RPDC en diciembre de 2011, tras la muerte de su padre. Educado en el extranjero y sin experiencia política ni militar, el joven Kim, de entonces 29 años, se convirtió en gobernante de un país sumido en la pobreza y el aislamiento internacional, pero con un programa nuclear en marcha.

Pese a que en un principio se especuló sobre la posibilidad de que Kim Jong-un implementara reformas para la apertura económica, parecidas a las de Deng Xiaoping en China, el nuevo dirigente norcoreano pronto hizo patente que continuaría privilegiando el desarrollo nuclear por encima de otras metas nacionales. Sus acciones iniciales también dejaron en claro que buscaría afianzar su poder mediante el reforzamiento del control sobre la población y la eliminación de sus opositores políticos. ${ }^{37}$ La llegada del tercer Kim tampoco cambió socialmente la historia: la Comisión de Naciones Unidas, para investigar los derechos humanos en Corea del Norte, difundió un reporte en marzo de 2014 en que documentaba "abusos atroces de amplio alcance en curso contra la población norcoreana", ${ }^{38}$ acusaciones que en su momento se habrían ya señalado en los casos del abuelo y el padre del actual Kim.

${ }^{37}$ El caso más sonado es la ejecución de su propio tío político y mentor, Jang Sung-taek, a finales de 2013. Jang, casado con una hermana del padre del actual líder, fue una de las figuras políticas y militares más importantes del país durante décadas, hasta que fue defenestrado por su sobrino, presuntamente por conspirar en su contra, entre otras faltas que van desde vender los recursos del país a un precio muy bajo hasta consumir drogas y cenar en restaurantes de lujo.

38 Oficina del Alto Comisionado de las Naciones Unidas para los Derechos Humanos, "North Korea: UN Commission documents wide-ranging and ongoing crimes against humanity, urges referral to ICC", Ginebra, 17 de febrero de 2014. 
En China, mientras tanto, el actual liderazgo, integrado por siete hombres que conforman el Comité Permanente del Buró Político del PCCh, con Xi Jinping a la cabeza, asumió el poder en noviembre de 2012. La prensa internacional ha subrayado las múltiples características que distinguen a $\mathrm{Xi}$ de los dirigentes chinos anteriores. ${ }^{39}$ Para este artículo basta con señalar que se tiene a Xi por el líder chino que más poder ha concentrado desde Mao Zedong y Deng Xiaoping y que es bien conocido por ser un fiel partidario de la línea reformista de este último.

Desde el inicio de su gestión, Xi ha asumido la dirección de una importante cantidad de ámbitos, sumados a la presidencia del país, de entre los que se destacan el ejército, la seguridad nacional y el proceso de reformas económicas actualmente en marcha. Asimismo, se han reforzado la vigilancia y las acciones de control sobre organizaciones no gubernamentales, activistas a favor de los derechos humanos y periodistas.

Simultáneamente, Xi ha instrumentado una muy severa campaña contra la corrupción en el Partido, las empresas estatales y el ejército, que ha derivado en que poco más de un millón de funcionarios hayan sido puestos bajo investigación por corrupción desde 2013 hasta septiembre de $2016 .{ }^{40}$ Gracias a esta campaña, Xi ha podido remover del camino a personajes contrarios a su liderazgo y consolidar su poder y visión de país.

En la esfera diplomática, la era Xi se ha caracterizado por el dinamismo chino en la escena internacional. China lo mismo se dedica a intentar construir una nueva arquitectura financiera mundial, que a proponer ambiciosas iniciativas económico-comerciales en Asia Central, Europa y el sudeste asiático. Igualmente envía cascos azules a diferentes puntos de África, que busca asumir el liderazgo de la lucha mundial contra el cambio climático.

Otra característica del nuevo líder chino es su acercamiento a Corea del Sur. A lo largo de su mandato, Xi Jinping se ha abocado

${ }^{39}$ Un bosquejo más completo del asunto puede encontrarse en la obra ya citada del exembajador en China J. E. Navarrete (pp. 22-24).

${ }^{40}$ Cf. L. Xin, "1 million officials probed for graft", Global Times, 24 de octubre de 2016, en http://www.globaltimes.cn/content/1013265.shtml, consultado el 20.III.2017. 
a mejorar la relación entre ambos países. Sorprende no poco que al realizar una elaborada visita de Estado a Seúl, donde se reunió en siete ocasiones con la presidente surcoreana Park Geun-hye en lo que va de 2012 a 2016, jamás se haya entrevistado con el líder norcoreano.

Queda aún por ver si, tras la destitución de la presidente Park en diciembre de 2016 por un escándalo de corrupción, el régimen chino continuará con la buena relación bilateral que ha tenido con Corea del Sur, lo cual dependerá primordialmente de la decisión que el nuevo gobierno surcoreano -cuando se elija- tome respecto a un espinoso tema: la instalación de una batería antimisiles estadounidense en su territorio, a la que China se opone tajantemente. En su momento, Park acordó con Estados Unidos el despliegue de un sistema de defensa antimisiles ante la amenaza que representa el programa de misiles norcoreano. El gobierno del presidente Xi ha argumentado que este sistema pondría en riesgo la seguridad nacional china y contribuiría a la desestabilización de la región.

También durante la gestión de Xi, China ha mostrado un mayor compromiso en lograr la desnuclearización de la península. Histórico ha sido el voto de China a favor del reforzamiento de las sanciones económicas, financieras y comerciales al régimen norcoreano, como lo han sido también las declaraciones oficiales de condena a los ensayos nucleares y de misiles, así como los llamados en los medios de comunicación, manejados desde el gobierno, a que Corea del Norte cese los mismos y retome la vía de la negociación para comenzar su desnuclearización.

Según se señaló anteriormente, Xi Jinping y Kim Jong-un aún no sostienen un encuentro bilateral, ni se han hecho una invitación a visitarse en sus respectivas capitales. Por esta situación, los contactos entre representantes de los respectivos gobiernos han sido escasos, salvo por la visita de Liu Yunshan, miembro del Comité Permanente del Buró Político del Comité Central del PCCh a Pyongyang en octubre de 2015, en ocasión del LXX Aniversario de la fundación del Partido de los Trabajadores Coreanos (PTC), durante la cual se reunió con Kim Jong-un, y la visita de Ri Su-yong, miembro del Buró Político del PTC y exministro de relaciones exteriores norcoreano a Beijing, ocasión en la que se reunió con el presidente Xi. 
Otro aspecto que generalmente denota el buen estado de las relaciones entre países comunistas es el que corresponde a los intercambios entre los respectivos partidos. En este caso, también esos contactos han disminuido sensiblemente, según lo evidencia la ausencia de un solo representante del PCCh en el VII Congreso del PTC, celebrado, por primera vez en treinta y seis años, en mayo de 2016.

La feroz ejecución de Jang Sung-taek, tío del actual líder norcoreano a finales de 2013, marcó un hito en la relación política entre ambos países. Jang no sólo era el enviado principal de Pyongyang para Beijing, sino un abierto defensor de las reformas económicas al estilo chino. Su ejecución, por encargo de su sobrino, ocurrió en medio de una purga de varios altos mandos del Partido, causando en el liderazgo político chino perturbación y dudas sobre el rumbo que tomará Kim.

\section{DE DIPLOMACIA Y SANCIONES}

El Río Tumen es una de las fronteras naturales entre China, Corea del Norte y Rusia. Justo en la triple frontera, en Hunchung, China, hay un mirador desde el que, con buen clima, pueden verse los dos países vecinos. Kilómetros río abajo, en el poblado de Tumen, hay un corredor turístico construido específicamente para atisbar uno de los países más misteriosos del orbe. Desde la ribera china se ven los campos de labranza de la aldea norcoreana de Namyang, edificios de viviendas, la garita migratoria e incluso un viejo tren de pasajeros, cuando éste llega procedente de Pyongyang. Los chinos han colocado estanquillos habilitados con binoculares en renta y locaciones para la foto del recuerdo, en los que además venden comida, bebidas y souvenirs; área de juegos infantiles con alberca; una plaza para espectáculos y hasta un pequeño muelle del que zarpan lanchas que se aproximan a la ribera opuesta, donde los campesinos trabajan antecedidos por dos minúsculas torres de vigilancia del ejército norcoreano. Donde se supondría hallar una de las fronteras más vigiladas del mundo, se encuentra una zona en entera calma. 
"No grite hacia la República Popular Democrática de Corea" es una de las instrucciones del gobierno chino a los paseantes, indicadas en letreros a lo largo del corredor turístico. Y es que la línea de agua que separa a ambos países a la altura de Tumen es tan delgada que las voces de un lado se escuchan en el otro y durante el verano podría cruzarse con el agua a las rodillas y en el invierno, caminando sobre el río congelado. Esta frontera trinacional es un sitio muy peculiar -étnica, lingüística y comercialmente-, donde mercancías, bienes diversos, monedas y personas coreanas, rusas y chinas se entrecruzan permanentemente por una estrecha franja de tierra y agua, sin muchos controles por parte de ninguno de los tres países.

Es difícil discernir los mecanismos precisos de toma de decisiones en materia de política exterior y de seguridad de cualquier país y, en particular, de China, donde los procesos se mantienen en su mayoría fuera del escrutinio público. Sin embargo, es esta aparente facilidad para cruzar la triple frontera ruso-sino-norcoreana, la que los especialistas en la relación bilateral destacan en la lista de razones por las cuales China evita a toda costa la desestabilización y colapso del régimen norcoreano. Beijing, dicen, siempre ha mirado con preocupación la posibilidad de oleadas de refugiados norcoreanos en su frontera norte y la presión que éstos generarían económica, demográfica y políticamente.

El otro punto fronterizo importante entre China y Corea del Norte es el río Yalu, que separa la ciudad china de Dandong de la norcoreana Sinuiju. Es ahí por donde, en tren, barco o camión, se realiza la mayor parte del comercio entre ambos países, lo que para Corea del Norte representa la mayor parte de su comercio con el mundo. A diferencia de Tumen, en Dandong puede verse una mayor presencia de fuerzas de seguridad en ambos lados, custodiando la frontera, pero no lo suficiente como para percibirse un ambiente de vigilancia permanente. Estas zonas fronterizas cobran mayor relevancia a la luz de las sanciones económicas impuestas por el Consejo de Seguridad de la onu a Corea del Norte, pues el $90 \%$ de los intercambios comerciales norcoreanos se realiza mediante Dandong o Tumen.

La discusión sobre la posible implementación de sanciones económicas multilaterales para presionar a la RDPC a frenar su 
programa nuclear inició a principios de la década de los años noventa, en el marco de la primera crisis nuclear norcoreana. La imposición de sanciones económicas multilaterales a cualquier país debe aprobarse en el Consejo de Seguridad, instancia donde, por años, China ha amenazado con hacer uso de su derecho de veto en caso de que alguna resolución -de las ocho que se aprobaron entre 1993 y 2003 condenando directa o indirectamente el programa nuclear y de misiles de Corea del Norte- implicara castigos de esa índole. Durante ese periodo, China se mantuvo firme en su postura, es decir que la única manera de resolver el tema es mediante la negociación y el diálogo, reiterando su escepticismo sobre la efectividad de las pretendidas sanciones. China decidió no vetar únicamente un par de resoluciones que imponían sanciones a las exportaciones e importaciones norcoreanas de armamento y bienes de lujo.

Justamente por eso es tan significativo el apoyo de China a la resolución 2270 del Consejo de Seguridad, adoptada en marzo de 2016, que establece un severo sistema de sanciones comerciales, financieras y marítimas sobre Corea del Norte. Pocos meses más tarde, el Consejo de Seguridad aprobó una nueva resolución, la 2321, que cierra aún más la pinza sobre Pyongyang, pues termina por prohibir las exportaciones norcoreanas de cobre, plata, níquel y zinc, por limitar las exportaciones de carbón, por pedir a los miembros de la onU que reduzcan el número funcionarios de las misiones diplomáticas y oficinas consulares de Corea del Norte (una de las principales fuentes de financiamiento del régimen) y por establecer más inspecciones de los cargamentos por mar, tierra o aire entre Corea del Norte y otros países, entre otras sanciones que se suman a las aprobadas en marzo del mismo año. Según el entonces secretario general de la ONU, el surcoreano Ban Kimoon, éste es el régimen de sanciones más severo que el Consejo de Seguridad haya impuesto desde su creación. ${ }^{41}$

41 Cf. Centro de Prensa de las Naciones Unidas, "Security Council Strengthens Sanctions on Democratic Republic of Korea, Unanimously Adopting Resolution 2321 (2016)", Nueva York, 30 de noviembre de 2016, en http://www.un. org/press/en/2016/sc12603.doc.htm, consultado el 1.I.2017. 
Además de la oposición a las sanciones que China mantuvo durante muchos años, su aproximación oficial al programa nuclear de la RDPC ha sido desde la diplomacia y se debe, en buena medida, gracias a que las gestiones chinas iniciaron, en 2003 en Beijing, las Conversaciones entre las Seis Partes, esquema de negociaciones en el que además de China y Corea del Norte participan Rusia, Estados Unidos, Japón y Corea del Sur.

Tras cuatro rondas de negociaciones, sostenidas todas en la capital china, las partes emitieron en 2006 un comunicado conjunto. En ese documento, Estados Unidos y Japón se comprometían a normalizar sus relaciones con Corea del Norte y a proporcionarle, junto con Rusia y Corea del Sur, suministros de energía. A cambio, Pyongyang detendría y desmantelaría su programa nuclear con fines bélicos y también regresaría al Tratado de No Proliferación Nuclear y permitiría el reingreso de inspectores de la AIEA.

Las conversaciones fueron abandonadas por Corea del Norte en 2009, alegando actitudes hostiles y dilaciones de parte de Estados Unidos. Desde el rompimiento, China ha instado a las partes a que regresen a la mesa de negociaciones. Estados Unidos y Corea del Sur han dicho que el requisito mínimo para retomarlas es que Corea del Norte abandone su programa nuclear. Kim Jong-un, de su parte, no se ha pronunciado en ningún sentido, pero a modo de respuesta ha continuado realizando ensayos nucleares y pruebas de misiles balísticos. Se estima que la falta de interés de Corea del Norte por retomar las conversaciones, junto con el reiterado alarde sobre los avances de su programa nuclear y de misiles, es lo que ha llevado a China a apoyar la imposición de sanciones y endurecer su discurso sobre Pyongyang.

Pese a todo esto, persisten fuertes dudas sobre la honestidad de la severidad china con respecto de las sanciones. Reportes recientes de algunos medios de comunicación internacionales sobre la dinámica en Dandong muestran que pese a las sanciones en vigor, el comercio entre ambos incluso se ha intensificado. ${ }^{42} \mathrm{Al}$ ser

42 Cf. L. Zhou y K. Huang, "Sanctions, What Sanctions? Inside the Chinese Border Town Doing Business with North Korea”, South China Morning Post, 3 de septiembre de 2016, en http://www.scmp.com/week-asia/article/2013134/ 
China el principal socio económico de Corea del Norte, su colaboración para la aplicación de las sanciones es fundamental. En otras palabras, el régimen de sanciones sólo tendrá éxito en la medida en que China lo aplique.

\section{Conclusiones}

La relación entre China y Corea del Norte atraviesa por una etapa de poca claridad. Cierto es que China se une a la condena internacional tras cada ensayo nuclear o de misiles efectuado por Corea del Norte. Cierto es también que ha votado a favor del endurecimiento del régimen internacional de sanciones y que ha insistido en la reanudación de las conversaciones entre las Seis Partes, que, de tener éxito, marcaría el fin del programa nuclear norcoreano. Sin embargo, China continúa siendo criticada por no tomar medidas más contundentes para lograr la desnuclearización y el desarme de un país sobre el que, se cree, conserva una gran influencia.

Si bien Beijing apoya la imposición de sanciones, el cumplimiento de las mismas se pone constantemente en duda, al revisar la actividad en la frontera sinocoreana. En otros espacios multilaterales, como el Consejo de Derechos Humanos, China bloquea iniciativas que buscan responsabilizar al gobierno norcoreano por violaciones de los derechos humanos de su población y se rehúsa también a asumir posturas enérgicas contra ese país, como cuando se documentó de manera suficiente que la RDPC estuvo detrás del hundimiento de un barco militar surcoreano en 2010, el cual dejó casi cincuenta marinos muertos. En general, China se conduce hacia Corea del Norte en el ámbito multilateral con una marcada ambivalencia, lo cual, sumado a la poca interacción -al menos oficial- entre sus élites políticas y partidistas, dificulta arribar a conclusiones claras sobre el estado de la relación bilateral.

Hay un consenso analítico según el cual uno de los mayores temores de China es el colapso paulatino del régimen norcoreano,

sanctions-what-sanctions-china-north-korea-and-dandong-deals, consultado el 16.XII.2016. 
que coadyuve a la unificación de la península bajo el control de Corea del Sur. Se argumenta esta razón, junto con la preocupación acerca del efecto desestabilizador de oleadas de norcoreanos que cruzan la frontera con China en busca de refugio, como los principales motivos del apoyo chino al gobierno de Pyongyang. China parece preferir la conservación del statu quo y contar con una nación amigable en la frontera noreste en lugar de correr el riesgo de presionar demasiado al régimen norcoreano, acelerando su colapso y posible reunificación con Corea del Sur, recreándose las temibles condiciones contra las que luchó hace más de sesenta años.

Sin embargo, con el paso del tiempo se ha ido haciendo evidente que la proliferación nuclear norcoreana tarde que temprano afectará también la seguridad y los planes de China en la región. Corea del Norte es hoy un Estado con armas nucleares, lo que puede propiciar que Japón y Corea del Sur busquen desarrollar su propio armamento nuclear y alteren el entorno de seguridad en el continente, cuya estabilidad es de suma importancia para los planes económicos de China.

China intenta balancear dos frentes: por un lado, su relación con un viejo camarada de armas a quien lo unen lazos históricos, una compleja red de intereses mutuos -entre éstos, evitar el colapso del régimen- $y$ una frontera que hay que mantener estable $y$, por otro, su intención de convertirse en uno de los principales actores internacionales que persiga la desnuclearización de la península, entre otras grandes metas multilaterales, y que mantenga buenas relaciones bilaterales con otros Estados.

Nuevos elementos colocan esta relación frente a mayores retos, que requerirán medidas más contundentes de parte de China. Uno de ellos es el marcado incremento de las acciones de provocación nuclear de Kim Jong-un. La última de éstas sucedió cuando el gobierno chino albergaba, a principios de septiembre de 2016, la XI Cumbre de Líderes del G20 en la sureña ciudad de Hangzhou. El 5 de septiembre, Pyongyang realizó tres pruebas de lanzamiento de misiles y, el 9 , su quinto y más potente ensayo nuclear (el segundo de 2016), cuyo efecto se sintió incluso en varias ciudades del noreste de China. Que Kim haya conducido un ensayo 
nuclear en su frontera con China, sin recato por el momento estelar de su aliado como anfitrión de la Cumbre, representó una afrenta similar a la del tercer ensayo, realizado por Kim en la víspera de la celebración del año nuevo chino en 2013, la fecha más importante y significativa en el calendario civil del país. Las pruebas nucleares del joven autócrata parecen estar convirtiéndose también en pruebas para la paciencia de la clase política china.

Hay que sumar ciertas peculiaridades de Kim Jong-un, quien además de haber asumido el poder sin credenciales comunistas ni experiencia en el manejo de asuntos de estado, funcionamiento partidista o relaciones internacionales, se comporta de una forma demasiado extravagante, voluble y poco diplomática para los gustos de la dirigencia china. Todo parece apuntar a que se acerca el momento en que China deberá replantear los términos de su relación con Corea del Norte, evaluando cuidadosamente los costos de continuar contribuyendo al mantenimiento del internacionalmente condenado régimen norcoreano o desmarcarse claramente del mismo, asumiendo los riesgos que su posible desfondamiento pueda acarrearle.

Hay señales que podrían estar indicando el rumbo que China tomará. Particularmente destaca, entre éstas, el anuncio, a mediados de febrero, de la suspensión total de las importaciones de carbón provenientes de la RPDC durante el resto de 2017 -una de las principales fuentes de los ingresos norcoreanos, como se revisó previamente-, al tiempo que el Canciller chino declaraba, durante su participación en la Conferencia de Seguridad de Munich, que las sanciones del CSONU a la RPDC se deben seguir implementando estrictamente.

Para la República Popular Democrática de Corea se percibe un riesgo mucho mayor en el actual estado de su relación con China. Pyongyang no puede permitirse perder a su único socio comercial y benefactor histórico. Pese a la conducta errática de Kim Jong-un, que tanto ha exasperado al gobierno chino, y la realización de ensayos nucleares en desafío a los reiterados llamados de China a la moderación, se estima poco probable que sea Pyongyang quien dé el primer paso para replantearse seriamente la relación con Beijing. 
La llegada de Donald Trump a la presidencia de Estados Unidos tiene un gran potencial para influir en la situación de la península norcoreana. Trump ha acusado reiteradamente a China de no apoyar a Estados Unidos para conseguir la desnuclearización de la RPDC y su secretario de Estado, Rex Tillerson, declaró durante su reciente visita a Corea del Sur, Japón y China, que Estados Unidos considera la acción militar para contener a la RPDC como una opción, dando por terminada la política de "paciencia estratégica” de la administración estadounidense anterior. Durante la breve estancia de Tillerson en China, Kim Jong-un aprovechó para manifestarse, realizando una prueba de motor para misiles de largo alcance, tecnología que le permitirá lanzar misiles de un continente a otro.

No queda claro qué capital diplomático podrá usar Trump para persuadir a China de endurecer su postura con Corea del Norte, considerando sus opiniones abiertamente críticas en contra de Beijing en diversos ámbitos, particularmente de carácter económico. Más aún, la implementación de una política exterior agresiva con China, como se prevé hará la nueva administración estadounidense, puede generar un efecto contrario al esperado, es decir que China busque reagrupar sus alianzas, al ser Corea del Norte una de las primeras en la fila.

De lo que no queda duda es que el rumbo que tome la relación sinonorcoreana continuará bajo el escrutinio internacional e involucrando a una gran cantidad de actores estatales y organismos internacionales. En un entorno mundial tenso como el actual, tanto el ascenso económico y diplomático de China, como la amenaza nuclear norcoreana, son elementos que contribuirán a configurar los años por venir.

\section{Bibliografía}

Albright, David, "North Korea Miniturization”, U.S.-Korea Institute, febrero de 2013, en http://38north.org/2013/albright021313

Anguiano, Eugenio (coord.), China contemporánea. La construcción de un país (desde 1949), México, El Colegio de México, 2011. 
Armstrong, Charles K., “«Fraternal Socialism»: The International Reconstruction of North Korea, 1953-1962”, Cold War History, vol. 5, núm. 2, 2005, pp. 161-187.

Cathcart, Adam y Charles Kraus, "The Bonds of Brotherhood: New Evidence on Sino-North Korean Exchanges, 1950-1954”, Journal of Cold War Studies, vol. 13, núm. 3, 2011, pp. 27-51.

Chanlett-Avery, Emma (coord.), "North Korea: U.S. Relations, Nuclear Diplomacy and Internal Situation”, Washington, DC, Congressional Research Service, 2016.

Centro de Prensa de las Naciones Unidas, "Security Council Strengthens Sanctions on Democratic Republic of Korea, Unanimously Adopting Resolution 2321 (2016)", Nueva York, 30 de noviembre de 2016, en http:/ / www.un.org/press/en/2016/sc12603.doc.htm

Chen, Jian, China's Road to Korean War: The Making of the Sino-American Confrontation, Nueva York, Columbia University Press, 1994.

Cheremukhin, Anton, Mikhail Golosov, Sergei Guriev y Aleh Tsyvinski, The Economy of People's Republic of China from 1953, National Bureau of Economic Research, 2015.

"China Brings Lavish Gifts to N. Korea", The Chosun Ilbo, 7 de octubre de 2009, en http://english.chosun.com/site/data/html_dir/2009/10/ 07/2009100700686.html

Comité Central del Partido Comunista de China, Textos escogidos de Deng Xiaoping 1975-1982, Beijing, Ediciones en Lenguas Extranjeras, 1984. ElBaradei, Mohamed, The Age of Deception. Nuclear Diplomacy in Treacherous Times, Londres, Metropolitan Books/Henry Holt \& Company, 2011.

Goncharov, Sergei N., John Lewis y Xue Litai, Uncertain Partners Stalin, Mao, and the Korean War, Stanford, University Press, 1993.

Haggard, Stephan y Marcus Noland, Famine in North Korea. Markets, Aid, and Reform, Nueva York, Columbia University Press, 2009.

- y Weiyi Shi, "Chinese Investment in North Korea: Some Data (Part I)", Peterson Institute for International Studies, junio de 2014, en https://piie.com/blogs/north-korea-witness-transformation/chinese-investment-north-korea-some-data-part-i

Hausmann, Ricardo, César A. Hidalgo, Sebastián Bustos, Michele Coscia, Sarah Chung, Juan Jiménez, Alexander Simoes y Muhammed A. Yildirim, The Atlas Of Economic Complexity. North Korea, Harvard's Center 
for International Development (CID), 2014, en http://atlas.media. mit.edu/en/profile/country/prk/\#dataviva

Hoom Kil, Soong y Chung-in Moon, Understanding Korean Politics: An Introduction, Albany, NY, State University of New York Press, 2001.

Jackson, Van, "Preventing Nuclear War with North Korea", Foreign Affairs, 11 de septiembre de 2016, en https://www.foreignaffairs.com/articles/north-korea/2016-09-11/preventing-nuclear-war-north-korea

Kim Il-Sung. Condensed Biography, Pyongyang, Foreign Languages Publishing House, 2001, p. 137.

Kissinger, Henry, On China, Nueva York, Penguin Press, 2011.

Lankov, Andrei, Crisis in North Korea: The Failure of De-Stalinization, 1956, Honolulu, University of Hawaii Press, 2004.

Nuclear Threat Initiative, "North Korea", reporte por país, octubre de 2016, en http://www.nti.org/learn/countries/north-korea/

Oficina del Alto Comisionado de las Naciones Unidas para los Derechos Humanos, "North Korea: UN Commission documents wide-ranging and ongoing crimes against humanity, urges referral to ICc", Ginebra, 17 de febrero de 2014.

Sanger, David E., "North Koreans Unveil New Plant for Nuclear Use", The New York Times, 20 de noviembre de 2010, en http://www.nytimes. com/2010/11/21/world/asia/21intel.html

Shen, Zhihua y Yafeng Xia, China and the Post-War Reconstruction of North Korea, 1953-1961, Washington, DC, Woodrow Wilson International Center for Scholars-North Korea International Documentation Project, 2012.

"Supreme Leader oversees successful SLBM test-fire", The Pyongyang Times, 27 de agosto, Juche 105, núm. 35, 2016, p. 1.

The World Fact Book, CiA, "North Korea 2015", octubre de 2016, en https://www.cia.gov/library/publications/the-world-factbook/ geos $/ \mathrm{kn} \cdot \mathrm{html}$

"Trading Economics 2015, North Korea”, septiembre de 2015, en http:// www.tradingeconomics.com/north-korea/gdp

Wilson Center, "Mao Zedong's Remarks at the Banquet for the North Korean Government Delegation”, 23 de noviembre de 1953, History and Public Policy Program Digital Archive, trad. de Jeffrey Wang y Charles Kraus, en http:/ /digitalarchive.wilsoncenter.org/document/ 114167 
, "Reference Materials for the Sino-Korean Negotiations on Supplying Equipment and Constructing Power Plants", 5 de agosto de 1958, History and Public Policy Program Digital Archive, trad. de Jeffrey Wang y Charles Kraus, en http://digitalarchive.wilsoncenter. org/document/114175

- "Journal of Soviet Ambassador in the DPRK A.M. Puzanov for 16 June 1960", 16 de junio de 1960, History and Public Policy Program Digital Archive, trad. de Gary Goldberg, en http:/ / digitalarchive.wilsoncenter.org/document/119419, consultado el 10.XII.2016.

World Nuclear Association, "Nuclear Proliferation Case Studies", septiembre de 2016, en http:/ /www.world-nuclear.org/information-library/ safety-and-security/non-proliferation/appendices/nuclear-proliferation-case-studies.aspx

Xin, Liu, "1 million officials probed for graft", Global Times, 24 de octubre de 2016, en http:/ /www.globaltimes.cn/content/1013265.shtml

Zhou, Laura y Kristin Huang, "Sanctions, What Sanctions? Inside the Chinese Border Town Doing Business with North Korea", South China Morning Post, 3 de septiembre de 2016, en http://www.scmp.com/ week-asia/article/2013134/sanctions-what-sanctions-china-north-korea-and-dandong-deals 